\title{
Nonlinear analysis of cycle slips in injection-locked oscillators
}

\author{
Sergio Sancho, Almudena Suarez, Franco Ramirez \\ University of Cantabria, D. of Communications Engineering. Santander, 39005, Spain
}

\begin{abstract}
A method is presented for the analysis of cycle slips in injection-locked oscillators. This nonlinear phenomenon gives rise to a temporal desynchronization between the injected oscillator and the input source due to noise perturbations. It involves very different time scales, so even envelope-transient based Monte Carlo analyses may suffer from high computational cost. The method presented is based on the extraction of a semianalytical nonlinear model of the injected oscillator. This reduced order model enables an efficient stochastic analysis of this oscillator, based on the use of the associated Fokker-Planck equation in the phase probability density. The analysis allows predicting the parameter-space regions that are best protected against cycle slips. The method has been applied to an injectionlocked oscillator at $5 \mathrm{GHz}$, with good agreement with commercial software simulations and measurements.

Index Terms - Cycle slips, injection-locked oscillators, nonlinear stochastic analysis, synchronization.
\end{abstract}

\section{INTRODUCTION}

Cycle slipping is a statistical nonlinear phenomenon that takes place in synchronized systems under the influence of noise. In the literature, it has been extensively analyzed in phase-locked loops (PLL) [1]-[3]. During cycle slips the oscillating system becomes unlocked from the reference source, which can cause a loss of data integrity in data communication systems, reducing the system ability to provide communications at an acceptable error rate.

In this paper, this phenomenon will be analyzed in microwave injection-locked oscillators. Since the occurrence of two cycle slips in a single stochastic realization can be separated in time by many thousands of reference cycles, the computational cost, even with envelope transient-based Monte Carlo analyses, would be very high in general. In the case of PLLs, this problem was solved in the seminal work [1] with the Fokker-Planck equation, used to predict the cycle slip probability by means of an eigenfunction expansion method [6]. In first and second-order PLL systems, the practical analysis in [1] was very efficient thanks to the reduced number $n$ of state variables involved (usually $n<3$ ).

In the case of the microwave injection-locked oscillators, a Fokker-Planck equation directly extracted from the system is unmanageable in practice, due to the high number of state variables involved. To circumvent this problem, in this paper a reduced-order semi-analytical model of the injected oscillator is extracted with Harmonic Balance (HB) simulations. This model was used in [4]-[5] to describe the deterministic transient dynamics of an injected oscillator. Unlike these previous works, here a stochastic analysis will be carried out. The reduced order of the semi-analytical differential equations will enable the extraction of a simple Fokker-Planck equation, which can be efficiently solved to predict the cycle slipping probability. This equation will be analyzed in terms of eigenfunctions and eigenvalues of the Fokker-Planck operator for an insightful study of the influence of the circuit parameters on the cycle slipping probability and transient dynamics under noise perturbations. The method has been applied to a microwave injection-locked oscillator a $5 \mathrm{GHz}$.

\section{STOCHASTIC ANALYSIS OF THE INJECTED OSCILLATOR}

The analysis will be carried out in the FET-based injectionlocked oscillator of Fig. 1. Using the technique in [4], the effect of all the noise sources existing in the circuit is modeled with an equivalent current generator $i_{n}(t)=\operatorname{Re}\left\{I_{n}(t) e^{j \omega_{i n} t}\right\}$ connected in parallel at the analysis node. Using the total admittance function at this observation node, the first-order perturbation model is [4]-[5]:

$$
Y\left(V_{0}+\Delta V(t), j \omega_{i n}+s, G_{i n} e^{j\left(\phi_{i n}-\phi\right)}\right)\left[V_{0}+\Delta V(t)\right] e^{j \phi(t)}=I_{n}(t)(1)
$$

where the synchronizing source (reference) is a voltage generator $v_{i n}(t)=\operatorname{Re}\left\{G_{i n} e^{j\left(\omega_{i n} t+\phi_{i n}\right)}\right\}, V_{0}$ is the node voltage amplitude in free-running conditions, $\Delta V(t)$ and $\phi(t)$ are, respectively, the increment in the node voltage amplitude and the node-voltage phase, $Y(V, j \omega)$ is the node current-to-voltage relationship at the first harmonic component and $s$ is the timederivative operator. Provided the input reference amplitude is small enough, equation (1) can be approached by a first-order Taylor series, which yields the following phase equation [4]:

$$
\dot{\varphi}=\Delta \omega+K \sin \varphi+\bar{H}^{+} \bar{n}(t) \equiv f(\varphi, \bar{n})
$$

where $f(\varphi, \bar{n})$ will be called the restoring term, $\Delta \omega=\omega_{0}-\omega_{i n}$ and the new state variable $\varphi=\phi+\alpha_{V}-\phi_{g}$ has been introduced. The terms $\alpha_{V}, \phi_{g}, K$ and $H$ are given by [4]:

$$
\begin{gathered}
Y_{V}=\left|Y_{V}\right| e^{j \alpha_{V}}, I_{g} e^{j \phi_{g}} \equiv-V_{0} Y_{g} G_{i n} e^{j \phi_{i n}}, I_{g} \in \mathbb{R}^{+} \\
K=\frac{I_{g}\left|Y_{V}\right|}{V_{0}\left(Y_{\omega}^{r} Y_{V}^{i}-Y_{\omega}^{i} Y_{V}^{r}\right)}, \bar{H}^{+}=\frac{\left[Y_{V}^{i}-Y_{V}^{r}\right.}{Y_{\omega}^{r} Y_{V}^{i}-Y_{\omega}^{i} Y_{V}^{r}}
\end{gathered}
$$

where $Y_{V}, Y_{\omega}$ and $Y_{g}$ stand for the derivatives of the totaladmittance function $Y$ of the free-running oscillator, calculated with respect to the corresponding variables: amplitude $V$, frequency $\omega$ and input reference value $G_{i n} e^{j \phi_{i n}}$. These derivatives can be numerically obtained through finite differences, introducing an auxiliary generator in the HB software [4]. The superindexes $r$ and $i$ stand for the real and imaginary parts, respectively. The vector 
$\bar{n}(t)=\left[I_{n}^{r}(t) I_{n}^{i}(t)\right]^{t} / V_{0}$ depends on the bandpass components of the equivalent noise current generator.

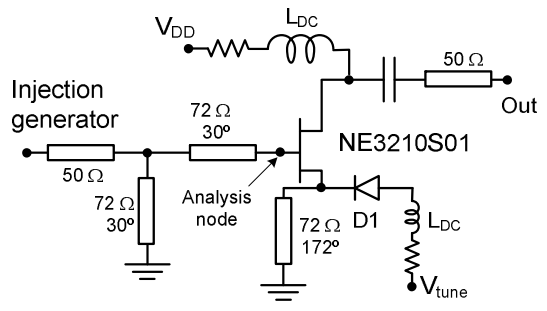

Fig. 1. Schematic of the injection-locked oscillator. The free-running frequency is $\mathrm{f}_{0}=5.185 \mathrm{GHz}$.

In the absence of noise sources, the steady-state solutions of (2) are time-constant synchronized solutions of node and saddle [7] type $\varphi_{n k}=\varphi_{n}+2 \pi k, \varphi_{s k}=\pi-\varphi_{n k}, k \in \mathbb{Z}$, with $\varphi_{n 0}, \varphi_{s 0} \in[0,2 \pi]$. These solutions coexist in the synchronization-frequency band, whose boundaries are given by $\Delta \omega_{L}= \pm|K|$. As $\Delta \omega$ approaches any of the two boundaries, both types of solutions get closer, eventually colliding in a saddle-node bifurcation at $\varphi_{n k}=\varphi_{s k}= \pm \pi / 2+2 \pi k$.

When noise sources are introduced into the circuit, the system trajectory fluctuates about a given stable steady-state solution $\varphi_{n k}$. Cycle slips may take place when the noise term inverts the polarity of the restoring term, such that sign $f(\varphi, \bar{n})=-\operatorname{sign} f(\varphi, \overline{0})$. If the polarity inversion extends over a long enough time interval, the trajectory is pushed towards the saddle solution $\varphi_{s k}$ along the interval $\left(\varphi_{n k}, \varphi_{s k}\right)$ (see Fig. 2). Once this solution is passed, the trajectory enters the stable manifold associated to $\varphi_{n, k \pm 1}$, evolving to this new solution. Although the new steady-state solution $\varphi_{n, k \pm 1}$ is simply phase shifted $2 \pi$ with respect to the previous one, during the transient the oscillator will be unlocked from the reference for a number of cycles determined by the transient time length. Such an event takes place with probability greater than zero provided that the variance of the noise process $\bar{n}(t)$ is high enough to invert the polarity of the restoring term.

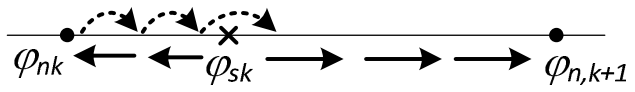

Fig. 2. Schematic of the cycle slipping mechanism. The timediscretized phase trajectory has been represented. Continuous arrows represent the trajectory in absence of noise, while dotted arrows represent the trajectory when noise inverts the restoring term polarity.

To analyze the effect of white noise Gaussian sources, the power spectral density of $\bar{n}(t)$ is set to $\mathrm{S}_{\mathrm{n}}=\Gamma$, where $\Gamma$ is a diagonal matrix whose values $\Gamma_{11}$ and $\Gamma_{22}$ are adjusted to fit the noise measurements of the free-running oscillator [4]. The capability of model (2) to predict the cycle slipping is demonstrated in Fig. 3, where a single time realization of the simulated phase process is represented. For comparison, the results of envelope transient in commercial $\mathrm{HB}$ have been superimposed. Both techniques predict a similar pattern of cycle slips in the time interval considered. During each jump, the oscillator remains unlocked and slips about one thousand reference periods. This transition time between two solutions is determined by the stability margin of the stable solution. Through perturbation analysis of equation (2), the stable pole is given by $v_{n}=K \cos \varphi_{n}<0$. Then, as the synchronization boundaries are approached, the magnitude $\left|v_{n}\right|$ decreases, increasing the transition time and the number of reference cycles slipped.

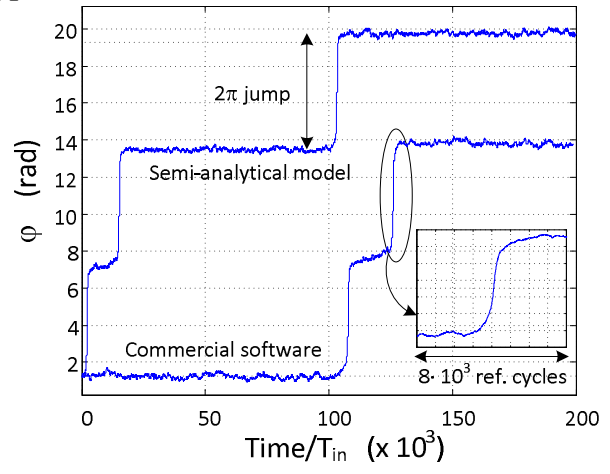

Fig. 3. Time realization of the phase process. Cycle slipping phenomenon for $f_{i n}=\omega_{i n} /(2 \pi)=1 / T_{i n}=5.18 \mathrm{GHz}$.

The cycle slipping probability within the synchronization band, will be efficiently analyzed with the Fokker-Planck equation [1],[5] associated to the stochastic differential equation (2):

$$
\begin{aligned}
& \frac{\partial p(\varphi, t)}{\partial t}=\left[-\frac{\partial}{\partial \varphi} k_{1}(\varphi)+\frac{k_{2}}{2} \frac{\partial^{2}}{\partial \varphi^{2}}\right] p(\varphi, t) \equiv L p(\varphi, t), \\
& k_{1}(\varphi)=K \sin \varphi+\Delta \omega, \quad k_{2}=\bar{H}^{+} \Gamma \bar{H}
\end{aligned}
$$

where $p(\varphi, t)$ is the time-varying probability density function (PDF) of the phase process $\varphi(t)$. The Fokker-Planck equation (4) can be solved by expanding the PDF in a basis of eigenfunctions of the operator $L$ as [1],[6]:

$$
p(\varphi, t)=\sum_{k=1}^{N} b_{k} e^{-\lambda_{k} t} p_{k}(\varphi), L p_{k}(\varphi)=-\lambda_{k} p_{k}(\varphi)
$$

where $b_{k}$ are coefficients determined by the initial condition $p(\varphi, 0)$. We will restrict the probability space to the interval $[0,4 \pi]$ and analyze the transitions between the two attractors $\varphi_{\mathrm{n} 0}$ and $\varphi_{\mathrm{n} 1}$. Periodic boundary conditions in the form $p(\varphi, t)=p(\varphi+4 \pi, t)$ will be assumed. Then, the eigenfunctions $p_{k}(\varphi)$ must be expressible in Fourier series as:

$$
p_{k}(\varphi)=\sum_{m=-M}^{M} c_{m k} \Psi_{m}(\varphi), \quad \Psi_{m}(\varphi) \equiv \exp \left(j m \frac{\varphi}{2}\right)
$$

Now, inserting expression (5) in equation (4) and using expansion (6), the following linear system is obtained [1]:

$$
A \bar{c}_{k}=\lambda_{k} \bar{c}_{k}, \quad 0=\lambda_{1}<\lambda_{N}^{r}<\cdots<\lambda_{N}^{r}, \quad A \equiv\left(A_{r m}\right)
$$

where the components of the matrix $A$ are given by the coefficients of the endomorphism $L$ in the basis $\left\{\Psi_{m}(\varphi)\right\}_{m}[1]$ 


$$
\begin{aligned}
& L \Psi_{m}(\varphi)=\sum_{r} A_{r m} \Psi_{r}(\varphi), \\
& A_{m \pm 2, m}=\mp K m / 4-1 / 2, \quad A_{m, m}=\left(-j 4 m \Delta \omega+m^{2} k_{2}\right) / 8
\end{aligned}
$$

where only the components $A_{m \pm 2, m}, A_{m, m}$ are different from zero. From (7), once the components $A_{r m}$ are obtained, the stochastic analysis reduces to the calculation of the eigenvalues and eigenvectors of the matrix $A$. The time evolution of the PDF $p(\varphi, t)$, associated to system (2), is represented in Fig. 4. The initial distribution is a Gaussian function centered at $\varphi_{n 0}$. As time evolves, there is a probability flow towards $\varphi_{n 1}$ due to the cycle slipping, until the steady state distribution $p(\varphi, t \rightarrow \infty)$ is reached. From (5), one can see that the steady-state distribution agrees with the eigenfunction $p_{0}(\varphi)$ associated to the eigenvalue $\lambda_{1}=0$.

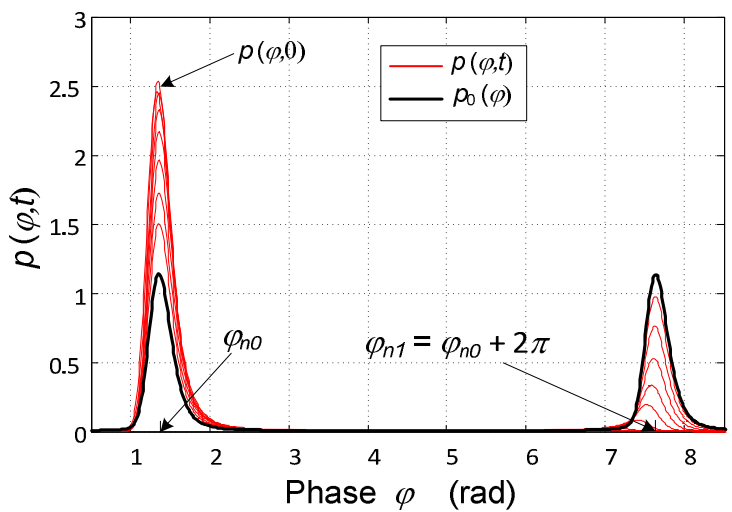

Fig. 4. Time evolution of the probability density function for $\mathrm{f}_{\mathrm{in}}=5.18$ $\mathrm{GHz}$, with $\Gamma_{11}=\Gamma_{22}=10^{-18}(\mathrm{~A} / \mathrm{V})^{2}$

Given an initial PDF distribution, the cycle slip probability is inversely proportional to the time $t_{s}$ needed to reach the steady state $p_{0}(\varphi)$. By inspection of (5),(7), it can be derived that $t_{s}$ decreases with $\lambda_{2}^{r}$, making the cycle-slip probability proportional to $\lambda_{2}^{r}$. This component has been traced in Fig. 5 versus $I_{Y g}=V_{0} Y_{g} G_{i n}$. In this figure, it is observed that as the boundaries $\Delta \omega_{L}= \pm|| K \mid$ of the synchronization range are approached, $\lambda_{2}^{r}$ increases, which leads to an increase of the cycle slipping probability. This is because in these regions the saddle and node solutions are close, reducing the size of the polarity-inversion interval. As gathered from Fig. 5, the cycle slipping phenomenon will limit the usable synchronization band. Fig. 5 also predicts the reduction of the cycle slipping probability as the reference amplitude $G_{i n}$ increases.

The results of Fig. 5 have been validated with measurements. In Figs. 6(a,b), the voltage waveform of the injection generator has been plotted versus the output voltage of the oscillator ( $\mathrm{X}-\mathrm{Y}$ mode) using an Agilent Infiniium 90000 Series Oscilloscope. Fig. 6(a) corresponds to $\Delta \omega=4 \pi \cdot 10^{6} \mathrm{rad} / \mathrm{s}$ and Fig. $6(\mathrm{~b})$ to $\Delta \omega=10 \pi \cdot 10^{6} \mathrm{rad} / \mathrm{s}$. In both measurements, most of the samples are on the noisy limit cycle. In Fig. 6(a), during cycle slips the oscillator is unlocked and some samples outside the cycle are obtained. This phenomenon occurs more often in Fig. 6(b), where $\Delta \omega$ has been increased towards the limit $\Delta \omega_{L}=|\mathrm{K}|$.

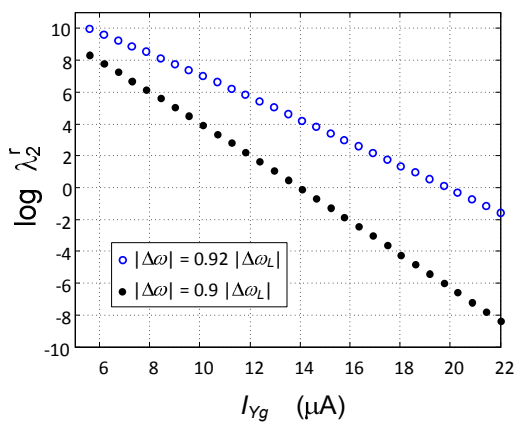

Fig. 5. Analysis of the cycle slipping probability. The cycle slips are more probable as $G_{i n}$ decreases or the border of the synchronization band is approached.

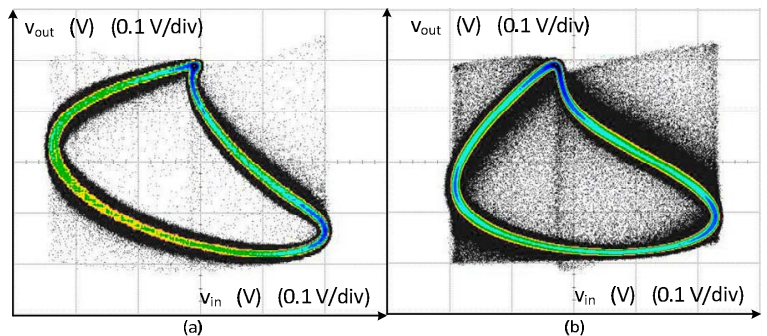

Fig. 6. Time domain measurements. Measuring time $T_{m}=10 \mathrm{~s}$. (a) $\Delta \omega=4 \pi \cdot 10^{6} \mathrm{rad} / \mathrm{s}$ (b) $\Delta \omega=10 \pi \cdot 10^{6} \mathrm{rad} / \mathrm{s}$

\section{CONCLUSIONS}

A method has been presented for the analysis of cycle slips in injection-locked oscillators. It relies on the analysis of the Fokker-Planck equation associated to a semi-analytical model of the system, which provides high computational efficiency. The method has allowed an investigation of the influence of the circuit parameters on the cycle slipping probability and the possible limitation of the usable synchronization band. It has been applied to an injected oscillator at $5 \mathrm{GHz}$ obtaining good agreement with simulations and measurements.

\section{ACKNOWLEDGEMENT}

This work was supported by the Spanish Ministry of Economy and Competitiveness under Contract TEC2011-29264-C03-01.

\section{REFERENCES}

[1] H. Meyr and G. Ascheid, Synchronization in Digital Communications, Wiley Inter-Science, New York, 1990

[2] U. L. Rohde, Microwave and Wireless Synthesizers: Theory and Design, Wiley Inter-Science, New York, 1997

[3] J. A. Crawford, Advanced Phase-Lock Techniques, Artech House, Boston, 2008

[4] F. Ramirez, M. Ponton, S. Sancho, A. Suarez, "Phase-Noise Analysis of Injection-Locked Oscillators and Analog Frequency Dividers," IEEE Transactions on Microwave Theory and Techniques, vol. 56, no.2, pp.393-407, Feb. 2008

[5] J. Dominguez, A. Suarez, S. Sancho, "Semi-analytical formulation for the analysis and reduction of injection-pulling in front-end oscillators," IEEE Microwave Symposium Digest, pp. 1589-1592, 7-12 June 2009

[6] H. Risken, The Fokker-Planck equation, Springer, New York, 1988

[7] S. Wiggins, Introduction to Applied Nonlinear Dynamical Systems and Chaos, Springer-Verlag, New York, 1990. 